\title{
Chronic liver disease and impaired hepatic glycogen metabolism in argininosuccinate lyase deficiency
}

\author{
Lindsay C. Burrage,, ${ }^{1,2}$ Simran Madan, ${ }^{1,3}$ Xiaohui Li, ${ }^{1}$ Saima Ali, ${ }^{1}$ Mahmoud Mohammad,,,5 \\ Bridget M. Stroup, ${ }^{1}$ Ming-Ming Jiang, ${ }^{1}$ Racel Cela, ${ }^{1}$ Terry Bertin, ${ }^{1}$ Zixue Jin, ${ }^{1}$ Jian Dai, ${ }^{6}$ Danielle \\ Guffey, ${ }^{7}$ Milton Finegold, ${ }^{8}$ Members of the Urea Cycle Disorders Consortium (UCDC), ${ }^{9}$ Sandesh \\ Nagamani, ${ }^{1,2}$ Charles C. Minard, ${ }^{7}$ Juan Marini, ${ }^{4,10}$ Prakash Masand, ${ }^{11}$ Deborah Schady, ${ }^{8}$ \\ Benjamin L. Shneider, ${ }^{2,12}$ Daniel H. Leung, ${ }^{2,12}$ Deeksha Bali, ${ }^{6}$ and Brendan Lee ${ }^{1,2}$ \\ 1Department of Molecular and Human Genetics, Baylor College of Medicine, Houston, Texas, USA. ${ }^{2}$ Texas Children's \\ Hospital, Houston, Texas, USA. ${ }^{3}$ Interdepartmental Program in Translational Biology and Molecular Medicine and ${ }^{4}$ USDA/ \\ ARS Children's Nutrition Research Center, Department of Pediatrics, Baylor College of Medicine, Houston, Texas, USA. \\ ${ }^{5}$ Department of Food Science and Nutrition, National Research Centre, Dokki, Giza, Egypt. ${ }^{6}$ Department of Pediatrics, Duke \\ Health, Durham, North Carolina, USA. ${ }^{7}$ Dan L. Duncan Institute for Clinical and Translational Research and ${ }^{8}$ Department \\ of Pathology, Baylor College of Medicine, Houston, Texas, USA. '95ee Supplemental Acknowledgments for Consortium \\ Details. ${ }^{10}$ Pediatric Critical Care Medicine, Department of Pediatrics, Baylor College of Medicine, Houston, Texas, USA. \\ ${ }^{11}$ Edward B. Singleton Department of Pediatric Radiology, Texas Children's Hospital, Houston, Texas, USA. ${ }^{12}$ Section of \\ Pediatric Gastroenterology, Hepatology, and Nutrition, Baylor College of Medicine, Houston, Texas, USA.
}

BACKGROUND. Liver disease in urea cycle disorders (UCDs) ranges from hepatomegaly and chronic hepatocellular injury to cirrhosis and end-stage liver disease. However, the prevalence and underlying mechanisms are unclear.

METHODS. We estimated the prevalence of chronic hepatocellular injury in UCDs using data from a multicenter, longitudinal, natural history study. We also used ultrasound with shear wave elastography and FibroTest to evaluate liver stiffness and markers of fibrosis in individuals with argininosuccinate lyase deficiency (ASLD), a disorder with high prevalence of elevated serum alanine aminotransferase (ALT). To understand the human observations, we evaluated the hepatic phenotype of the $A s /^{\text {Neo/Neo }}$ mouse model of ASLD.

RESULTS. We demonstrate a high prevalence of elevated ALT in ASLD (37\%). Hyperammonemia and use of nitrogen-scavenging agents, 2 markers of disease severity, were significantly $(P<0.001$ and $P$ $=0.001$, respectively) associated with elevated ALT in ASLD. In addition, ultrasound with shear wave elastography and FibroTest revealed increased echogenicity and liver stiffness, even in individuals with ASLD and normal aminotransferases. The $A s /^{\mathrm{Neo} / \mathrm{NeO}}$ mice mimic the human disorder with hepatomegaly, elevated aminotransferases, and excessive hepatic glycogen noted before death (3-5 weeks of age). This excessive hepatic glycogen is associated with impaired hepatic glycogenolysis and decreased glycogen phosphorylase and is rescued with helper-dependent adenovirus expressing As/ using a liverspecific (ApoE) promoter.

co-first authors.

Conflict of interest: The authors have declared that no conflict of interest exists.

Copyright: ( 2020 , American Society for Clinical Investigation.

Submitted: September 3, 2019

Accepted: January 15, 2020

Published: February 27, 2020.

Reference information: /CI Insight. 2020;5(4):e132342.

https://doi.org/10.1172/jici.

insight.132342.

CONCLUSION. Our results link urea cycle dysfunction and impaired hepatic glucose metabolism and identify a mouse model of liver disease in the setting of urea cycle dysfunction.

TRIAL REGISTRATION. This study has been registered at ClinicalTrials.gov (NCT03721367, NCT00237315).

FUNDING. Funding was provided by NIH, Burroughs Wellcome Fund, NUCDF, Genzyme/ACMG Foundation, and CPRIT. 


\section{Introduction}

Argininosuccinic aciduria (also referred to as argininosuccinate lyase deficiency; ASLD) (MIM 207900) is an autosomal recessive disorder caused by a deficiency of argininosuccinate lyase (ASL) (1). ASL is an enzyme in the urea cycle that converts argininosuccinic acid (ASA) to arginine and fumarate. ASLD is characterized by arginine deficiency and accumulation of ASA, the diagnostic metabolite, in plasma and urine. In addition to this catalytic function, ASL has a structural role as a component of a protein complex with nitric oxide synthase (NOS) that channels extracellular arginine to NOS for nitric oxide (NO) production (2). Thus, individuals with ASLD may have both urea cycle dysfunction with arginine deficiency from decreased catalytic activity and NO deficiency if the structural integrity of the protein complex required for the synthesis of NO is also deficient $(2,3)$. In the early-onset form of ASLD, individuals present with neonatal hyperammonemia, whereas individuals with the late-onset form of the disorder may present with hyperammonemia in the setting of stress at a later age or may never have episodes of hyperammonemia. Other clinical features of the disorder may include trichorrhexis nodosa, cognitive impairment, seizures, hypokalemia, diarrhea, and liver disease (4). Systemic hypertension has also been described in a subset of individuals with $\operatorname{ASLD}(3,5-8)$. Treatment typically includes low-protein diet with essential amino acid supplementation, arginine supplementation, use of nitrogen-scavenging agents, and — in some cases — liver transplantation.

Preliminary analysis performed over 10 years ago using the baseline, cross-sectional data from the Longitudinal Study of Urea Cycle Disorders, a natural history study performed by the UCDC of the Rare Diseases Clinical Research Network (RDCRN) suggested that ASLD may be associated with a heavier burden of hepatic complications as compared with other urea cycle disorders (UCDs) (9). Liver complications have been observed in both early-onset and late-onset forms of ASLD and in patients treated with standard therapies (4). These complications in ASLD may be demonstrated by elevated transaminases, abnormal liver echogenicity on ultrasound, hepatomegaly, hepatic fibrosis, cirrhosis with portal hypertension, and impaired synthetic function, which - in some patients - has necessitated liver transplantation (10-20). Common liver histopathologic findings in patients with ASLD and other UCDs include hepatocyte enlargement with pallor, increased glycogen deposition, steatosis, fibrosis, and nodularity $(10-12,15,20-22)$. Lastly, rare cases of hepatocellular carcinoma have also been described in UCDs including 1 individual with $\operatorname{ASLD}(4,23-25)$.

In this study, we analyzed the data from the Longitudinal Study of Urea Cycle Disorders performed by the UCDC to assess the impact of chronic hepatocellular injury in a large population of individuals with various UCDs, including ASLD. We used these same data to determine factors associated with increased risk for chronic hepatocellular injury in ASLD. However, we hypothesize that chronic elevations of alanine aminotransferase (ALT) and aspartate aminotransferase (AST) may not identify all individuals with chronic liver disease in this disorder. To test this hypothesis in a proof-of-principle study, we used additional noninvasive assessments (ultrasound, shear wave elastography [SWE], and serum biomarkers of fibrosis) to evaluate for evidence of liver disease in 8 participants with ASLD, some of whom have normal levels of ALT and AST. Lastly, we explore the hepatic phenotype and potential therapies in a hypomorphic mouse model of ASLD ( $A s t^{\text {tmlBrle }}$; referred to hereafter as $A s l^{\text {Neo/Neo }}$ ), which has $\sim 16 \%$ residual enzyme activity and recapitulates many other phenotypic features of human $\operatorname{ASLD}(2,3,26,27)$.

\section{Results}

Chronic hepatocellular injury in ASLD. Among 640 total participants in the Longitudinal Study of Urea Cycle Disorders (28), a total of 2978 ALT levels and 2979 AST levels were available. The number of ALT or AST levels per individual varied from 0-19 values. Two or more serum ALT and/or AST levels were available for 494 participants (77\%; mean age, 17 years; range, 0.01-71.5 years). For each disorder, we determined the percentage of participants with $\geq 2$ ALT or AST levels above $100 \mathrm{U} / \mathrm{L}, 150 \mathrm{U} / \mathrm{L}$, or $200 \mathrm{U} / \mathrm{L}$ (Figure 1). A threshold of $100 \mathrm{U} / \mathrm{L}$ was selected as a marker for chronic hepatocellular injury because this level is approximately 2 or more times higher than the upper limit of normal for most age ranges at most laboratories. Overall, $11 \%(n=55)$ of participants with a UCD had $\geq 2$ ALT levels above $100 \mathrm{U} / \mathrm{L}$ at routine study visits, and $5 \%(n=27)$ of participants with a UCD had $\geq 2$ AST levels above $100 \mathrm{U} / \mathrm{L}$. Likewise, $7 \%(n=$ 34) of participants had $\geq 2$ ALT levels above $150 \mathrm{U} / \mathrm{L}$ at routine study visits, and $3 \%(n=15)$ of participants had $\geq 2$ AST levels above $150 \mathrm{U} / \mathrm{L}$. Using the highest threshold, only $4 \%$ ( $n=19$ participants) had $\geq$ 2 ALT levels above $200 \mathrm{U} / \mathrm{L}$, and $1 \%(n=6)$ of participants had $\geq 2$ AST levels above $200 \mathrm{U} / \mathrm{L}$. Regardless of the threshold used, the disorders affecting the most distal enzymes in the urea cycle, ASLD $(n=82)$ and 

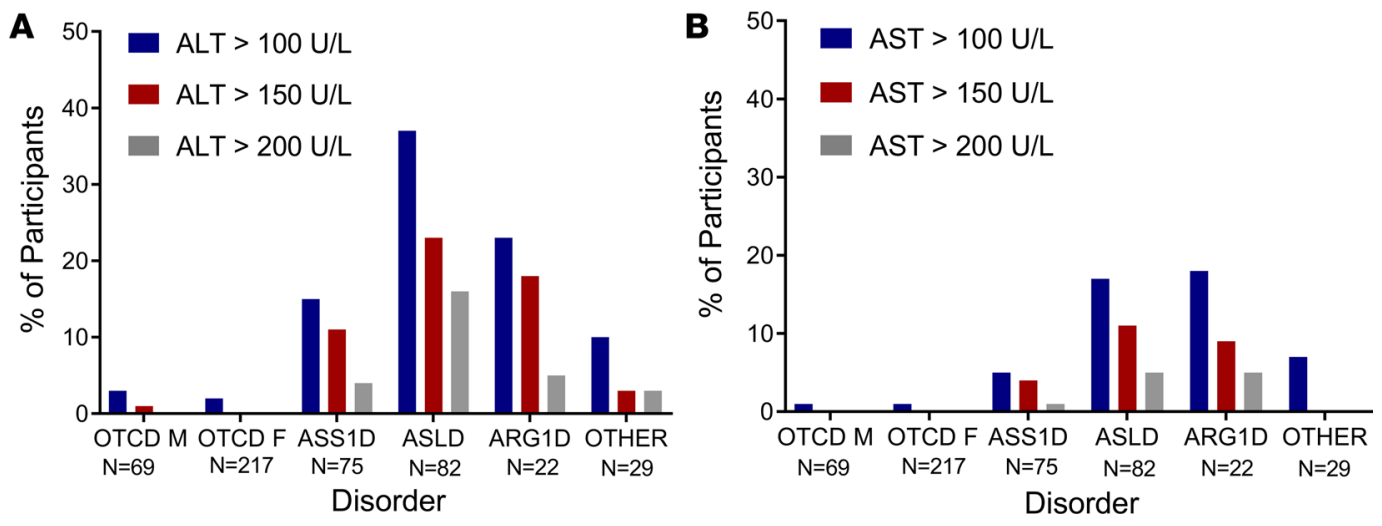

Figure 1. Increased aminotransferase levels in individuals with UCDs. (A) The percentage of participants with each disorder who had 2 or more ALT levels above the indicated threshold are shown. (B) The percentage of participants with each disorder who had 2 or more AST levels above the indicated threshold are shown. ALT, alanine aminotransferase; AST, aspartate aminotransferase; OTCD, ornithine transcarbamylase deficiency; ASS1D, argininosuccinate synthetase deficiency; ASLD, argininosuccinate lyase deficiency; ARG1D, arginase deficiency; OTHER, other urea cycle disorders; $M$, male, $F$, female.

arginase deficiency (ARG1D, $n=22$ ), had a high prevalence of chronic hepatocellular injury. For instance, for $\geq 2$ ALT levels above $100 \mathrm{U} / \mathrm{L}$, the prevalence was $37 \%$ and $23 \%$ for ASLD and ARG1D, respectively (Figure 1A). Using this same threshold for ALT, the prevalence of chronic hepatocellular injury for males and females with ornithine transcarbamylase deficiency (OTCD) was only $2 \%-3 \%$ in both groups. For citrullinemia (ASS1D, $n=75$ ), $15 \%$ of participants had $\geq 2$ ALT levels above $100 \mathrm{U} / \mathrm{L}$ (Figure 1A).

To compare disorders directly, we used a general linear mixed model. For both AST and ALT, the effect of time was independent of the disorder. Thus, the interaction term between disorder and time was excluded for the final model. For ALT, there were significant differences in ALT values between disorders $(P<0.001)$ and baseline age $(P<0.001)$. Of note, ALT decreases by $0.8 \%$ per year for baseline age. Moreover, individuals with ASLD who were identified to have chronic hepatocellular injury included infants, children, and adults. When comparing disorders (Supplemental Table 1; supplemental material available online with this article; https://doi.org/10.1172/jci.insight.132342DS1), ALT was higher among participants with ASLD as compared with males with OTCD $(P<0.001)$, females with OTCD $(P<0.001)$, and individuals with ASS1D $(P<0.001)$. ALT was not significantly different between individuals with ASLD versus ARG1D. Similar results were obtained with analysis of AST (Supplemental Table 1). Given the sample size and high prevalence of chronic hepatocellular injury in ASLD (as assessed by ALT), we conducted detailed studies in a subset of individuals with ASLD, as well as in a mouse model of the disorder, in order to further explore mechanisms contributing to hepatic disease.

Chronic hepatocellular injury in ASLD is associated with disease severity. Of the covariates tested, younger age, history of hyperammonemia, and use of nitrogen scavenging agents were significantly associated with ALT and AST levels (Table 1). The finding of an association with younger age is not surprising, as the normal ranges for ALT and AST in very young children are wider than the normal ranges in older children and adults. However, the finding of an association with history of hyperammonemia and use of nitrogen-scavenging agents suggests that individuals with more severe forms of ASLD associated with hyperammonemia and need for a nitrogen-scavenging agent are at increased risk for chronic hepatocellular injury. Molecular data were available for 24 participants with ASLD, and more than 1 ALT or AST level was available in 23 of these 24 participants with molecular data (Supplemental Table 2). Of these 23 participants, 7 (30\%) had 2 or more ALT or AST greater than $100 \mathrm{U} / \mathrm{L}$, and all of these participants had a history of hyperammonemia. All of these 7 participants had at least 1 variant that was previously associated with severe disease (29). Moreover, of the 5 participants with 2 severe alleles, 4 had evidence of chronic hepatocellular injury using our definition. Thus, the molecular data further support the results from the linear regression model, which suggests that severe disease is associated with a higher prevalence of chronic hepatocellular injury.

Elevated international normalized ratio in UCDs. Similarly, we determined the percentage of participants with 2 or more international normalized ratio (INR) values $\geq 1.2$ or $\geq 1.3$ as a measure of liver function. Two or more INR values were available for 395 participants (62\%; mean age, 18 years; range, $0.01-71.5$ years). 
Table 1. Linear regression analysis reveals covariates associated with aminotransferase levels in individuals with ASLD

\begin{tabular}{|c|c|c|c|c|}
\hline Covariate & $P$ value (ALT) & $R^{2}$ & $P$ value (AST) & $\boldsymbol{R}^{2}$ \\
\hline Age & 0.002 & 0.087 & $<0.001$ & 0.2008 \\
\hline Sex & 0.979 & 0.0003 & 0.741 & 0.0046 \\
\hline Hyperammonemia & $<0.001$ & 0.5018 & $<0.001$ & 0.2776 \\
\hline Plasma citrulline (baseline) & 0.016 & 0.0367 & 0.069 & 0.0255 \\
\hline Plasma arginine (baseline) & 0.200 & 0.0031 & 0.525 & 0.0044 \\
\hline Plasma glutamine (baseline) & 0.505 & 0.0003 & 0.684 & 0.0046 \\
\hline Phenylbutyrate & $<0.001$ & 0.2707 & $<0.001$ & 0.3160 \\
\hline Benzoate & 0.001 & 0.1122 & 0.001 & 0.1150 \\
\hline Either phenylbutyrate or benzoate & 0.001 & 0.1099 & $<0.001$ & 0.1623 \\
\hline High-dose arginine & 0.363 & 0.0002 & 0.761 & 0.0046 \\
\hline
\end{tabular}

Linear regression analysis reveals that age, presence of 1 or more episodes of hyperammonemia, use of phenylbutyrate-containing medications, use of sodium benzoate, or use of either phenylbutyrate-containing medications or sodium benzoate are associated with ALT and AST levels. There is a trend suggesting that baseline citrulline may also be associated with ALT and AST levels, but this association fell short of significance for AST.

Of these 395 subjects, $20 \%(n=78)$ had 2 or more INR values $\geq 1.2$ and $5 \%(n=20)$ had 2 or more INR values $\geq 1$. Sixty-eight percent of participants with ARG1D had 2 or more INR values $\geq 1.2$, whereas $27 \%$ of participants with ASLD had 2 or more INR values $\geq 1.2$. The percentage of subjects who had 2 or more INR values $\geq 1.2$ with ASS1D, OTCD (males), and OTCD (females) was 20\%, 21\%, and 11\%, respectively. When using the higher threshold, $32 \%$ of individuals with ARG1D had 2 or more INR values $\geq 1.3$. Fewer than $10 \%$ of participants with the other disorders had INR values above the higher threshold (Supplemental Figure 1 and Supplemental Table 1).

Hepatic explants from individuals with ASLD show fibrosis and hepatic glycogen accumulation. Hepatic biopsies are not routinely performed in individuals with UCDs. Moreover, explant tissue and liver biopsy tissue are not routinely collected as part of the Longitudinal Study of Urea Cycle Disorders. Thus, to investigate whether serum levels of ALT or AST correlate with hepatic pathology in ASLD, we performed a retrospective review of the histopathology of all liver explants, liver biopsies, or liver autopsy samples from individuals with UCDs ( $n=29$; age range, 6 days to 8 years, 2 months) from January 1, 1995, to July 1, 2016, at Texas Children's Hospital, one of the lead sites for the UCDC. Samples were available from a total of 29 individuals with UCDs, and 4 of these individuals had ASLD (14\%). Most available samples ( $n=16,55 \%)$ were from male infants with OTCD. Remaining samples were from females with OTCD $(n=3,10 \%), 1$ with hyperornithinemia-hyperammonemia-homocitrullinuria syndrome $(\mathrm{HHH}), 3$ with CPS1 deficiency, and 2 with ARG1D.

Three of the 4 liver explants from individuals with ASLD had advanced fibrosis or cirrhosis (stage 3 or 4), and 1 explant had mild periportal fibrosis (stage 1) (Figure 2, A and B, and Supplemental Table 3; ref. 30). At the time of transplant, all 4 individuals with ASLD had elevations in ALT and/or AST, and 2 individuals were known to have liver fibrosis (stage 3-4) by liver biopsy performed within the 6 months before the transplantation. All 4 explants had marked cytoplasmic glycogenosis qualitatively by periodic acid-Schiff (PAS) stain with and without diastase (Figure 2, C and D, and Supplemental Figure 2). Of the other 25 samples, only 1 (male, OTCD, 8 months of age) had stage 3 or stage 4 fibrosis. Although hepatic glycogen accumulation was a common finding in explant tissue from individuals with UCDs, hypoglycemia is not a typical feature of these disorders.

Noninvasive biomarkers of fibrosis in ASLD. We hypothesized that assessing liver disease based on serum levels of ALT or AST is not sufficiently sensitive to identify all individuals with ASLD who have significant liver disease. To test this hypothesis, we performed traditional grayscale ultrasound and SWE to assess liver stiffness, and we validated serum biomarkers for fibrosis (e.g., APRI and FibroTest) in 8 participants with ASLD as part of a larger study of liver disease in UCDs (Table 2). All participants had normal ammonia levels and were at baseline state of health at the time of study participation. Of these 8 participants (age range, 5-31 years; BMI range, $\left.16-26 \mathrm{~kg} / \mathrm{m}^{2}\right), 2(25 \%)$ had coarse echogenicity or diffuse increased echogenicity on ultrasound. By SWE, all 8 scans were valid with an IQR/median under 0.3 , and 5 participants (63\%) had elevated liver stiffness ( $\mathrm{F}>0$, median liver shear wave speed $\geq 1.35$ ). In addition, 3 participants $(38 \%)$ had elevated FibroTest ( $n=2 \mathrm{~F} 1, n=1 \mathrm{~F} 1-\mathrm{F} 2)$. Overall, 2 participants $(25 \%)$ had increased echogenicity on 
A

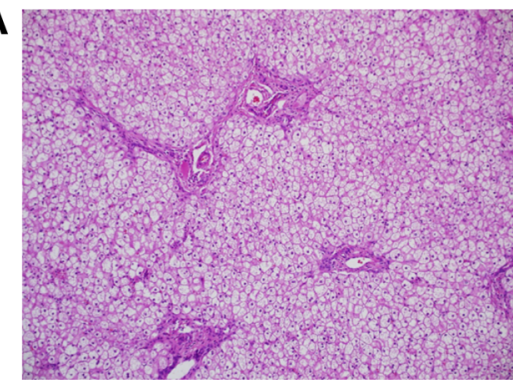

C

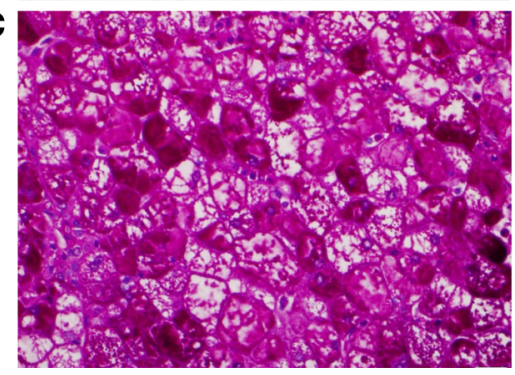

B

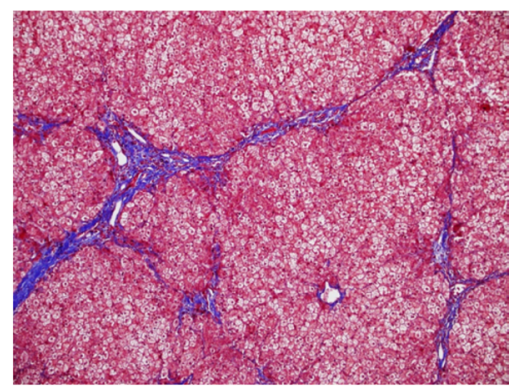

D

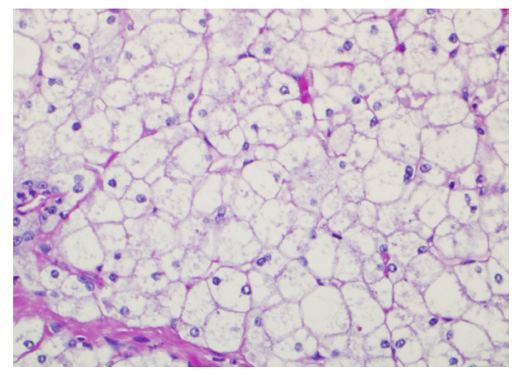

Figure 2. Hepatic fibrosis in ASLD. (A) H\&E stain of section of liver explant from a 20-month-old male with ASLD shows periportal fibrosis (stage 1; original magnification, 100x). Hepatocytes are swollen. (B) Trichrome stain of section of liver explant from a 2-year, 7-month-old female with ASLD shows diffuse bridging fibrosis (stage 3; original magnification, 10x). Hepatocytes are swollen. (C) PAS stain (without diastase) of section of liver explant from 5-year, 11-month-old female with ASLD and stage 4 fibrosis (original magnification, 400x). (D) PAS stain with diastase of section of liver explant from the same individual as in C demonstrates absence of PAS stain, which indicates that the PAS-stained material, in the absence of diastase, is glycogen (original magnification, 400x).

ultrasound, increased liver stiffness, and increased FibroTest, despite having normal ALT and AST on the day of their exams. Of note, the FibroTest results were not significantly correlated with BMI, age, AST, ALT, or liver stiffness. This result suggests that serum aminotransferase levels alone may not identify individuals with ASLD who have liver disease.

$A s^{\text {Neo/Neo }}$ mice have chronic hepatocellular injury. To assess whether the hypomorphic $A s^{\text {Neo/Neo }}$ mouse model of ASLD recapitulates the hepatocellular injury and other hepatic complications observed in patients, the hepatic phenotype of the $A s^{\text {Neo/Neo }}$ mice was analyzed in 3- to 4-week-old mice before weaning. This young age was selected for analysis, as the $A s^{\text {Neo/Neo }}$ mice typically do not survive weaning and die from multiorgan dysfunction (2). As previously demonstrated, plasma ALT and AST were significantly elevated in $A s l^{\text {Neo/Neo }}$ mice as compared with WT littermates, whereas direct bilirubin and albumin were not significantly different (Figure 3, A and B, Supplemental Figure 3, and refs. 2, 26, 27). In addition, the $A s^{\text {Neo/Neo }}$ mice had hepatomegaly as compared with WT littermates (Figure 3C). At this age, the mice did not show evidence of hepatic fibrosis or cirrhosis. Electron microscopy of the sections of the liver from an $A s l^{\text {Neo/Neo }}$ versus WT mouse revealed enlarged, swollen hepatocytes with increased cytoplasmic hepatic glycogen causing displacement of the organelles toward the cell membrane in the $A s l^{\text {Neo } / N e o}$ mouse (Figure $3 \mathrm{D}$ and Supplemental Figure 2). Moreover, the hepatic glycogen in the $A$ s $^{\text {Neo/Neo }}$ mouse appeared to be more consistent with $\beta$-particles, whereas the glycogen in the WT sample was more consistent with the $\alpha$-rosette configuration (Supplemental Figure 2). Quantification of hepatic glycogen in 3- to 4-hour fasted

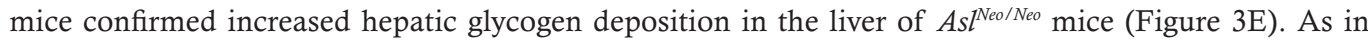
individuals with ASLD, therapy with sodium benzoate and arginine (provided in the drinking water, as previously described) to decrease hyperammonemia and to prevent systemic arginine deficiency (2) did not result in improvement in the hepatocellular injury phenotype (Supplemental Figure 4).

Decreased hepatic glycogenolysis in Asl ${ }^{\text {Neo/Neo }}$ mice. To evaluate hepatic glycogenolysis in the Asl ${ }^{\text {Neo/Neo }}$ mice,

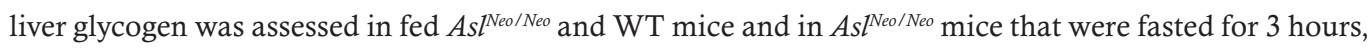
12 hours, or 24 hours. Although the hepatic glycogen content was similar in the fed state, $A s^{\text {Neoo Neo }}$ mice had higher hepatic glycogen content after 3-hour, 12-hour, and 24-hour fasts, suggesting that hepatic glycogenolysis is impaired in the $A s^{\text {INoo Neo }}$ mice (Figure $4 \mathrm{~A}$ ). Moreover, the decline in hepatic glycogen during the fast was much slower in $A s^{\mathrm{Neo} / \mathrm{N} N o}$ mice. To further assess glycogen metabolism, the hepatic 
Table 2. Biomarkers for liver disease in study participants with ASLD

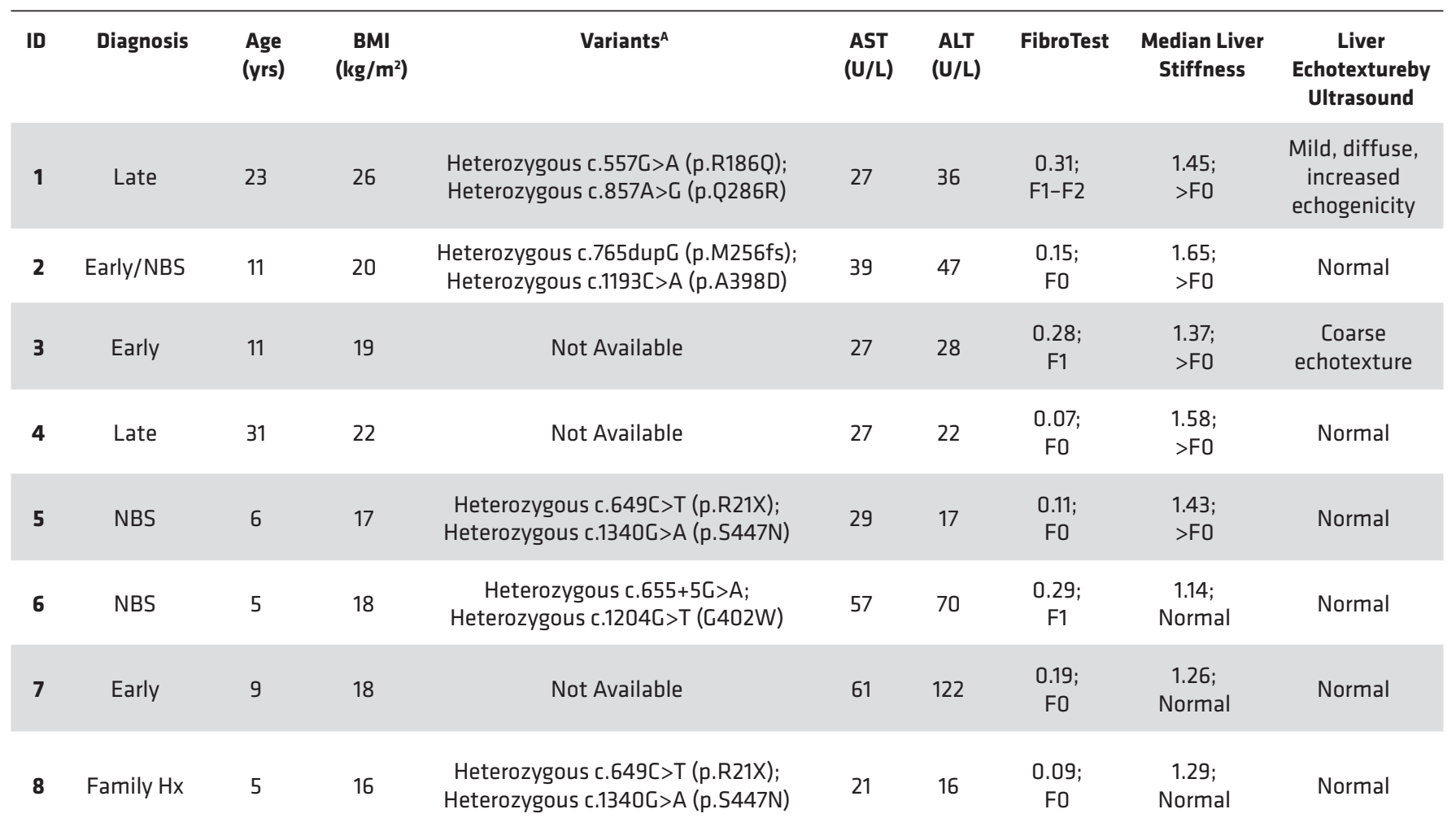

Data for study participants enrolled in the biomarker study is provided. Late, late onset; Early, early onset (within the first month of life); NBS, newborn screening; Family Hx, family history; yrs, years. ${ }^{A}$ For variants, NM_001024943.1 is the transcript used.

enzyme activities of glucose-6-phosphatase, glycogen phosphorylase, branching enzyme, debranching enzyme, and phosphorylase kinase were measured. The activity of all enzymes except glycogen phosphorylase were similar in the liver of $A s l^{\text {Neo/Neo }}$ versus WT mice (Supplemental Figure 5 and Figure 4B). In contrast, the activity of the hepatic glycogen phosphorylase, the enzyme that catalyzes the rate-limiting step of glycogenolysis, was significantly lower in $A s l^{\text {Neo } / N e o}$ mice. This $\sim 35 \%$ reduction in hepatic glycogen phosphorylase activity was not due to reduced RNA expression (Figure 4C). However, protein expres-

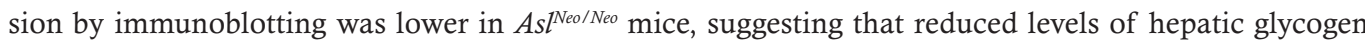
phosphorylase protein may explain the reduction in enzyme activity (Figure 4D).

To further evaluate glucose metabolism in this mouse model, we assessed plasma glucose levels. After a 12-hour fast, the plasma glucose levels were similar in the 2 groups (Figure $5 \mathrm{~A}$ ). We also assessed plasma glucagon levels. Measures of plasma glucagon after a 3-hour fast were similar in $A s^{\text {Neo/Neo }}$ versus WT mice, with a trend suggestive of possible increased glucagon after a 24-hour fast in $\mathrm{Asl}^{\mathrm{Neo} / \mathrm{Neo}}$ mice (Figure 5B).

Hepatic glycogen synthesis is similar in Asl ${ }^{\text {Neo/Neo }}$ mice. We next assessed hepatic glycogen synthesis after a

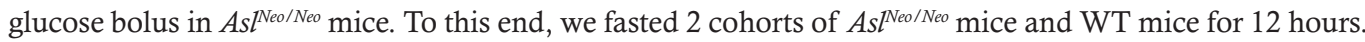
Then, we collected plasma for glucose measurements and liver samples for hepatic glycogen quantification in 1 cohort of mice after the fast and provided a glucose bolus to the second cohort of mice. Blood glucose after the fast was not significantly different in the 2 groups. Two hours after the bolus, we collected liver samples for hepatic glycogen quantification from the second cohort of mice. At baseline, we confirmed significantly increased hepatic glycogen accumulation in $A s l^{\text {Neo/Neo }}$ mice versus WT mice, with a similar magnitude of increase after the bolus (Figure 5C). Thus, glycogen synthesis in response to a glucose bolus does not appear to be different in the $A s l^{\text {INo/Neo }}$ mice versus WT mice.

Helper-dependent adenovirus expressing Asl rescues hepatic phenotype. We next tested whether the hepatocellular injury and increased hepatic glycogen deposition with reduced hepatic glycogen phosphorylase activity could be rescued with hepatocyte-specific expression of $A s l$. To this end, $A l^{\text {Neo/Neo }}$ and WT mice 

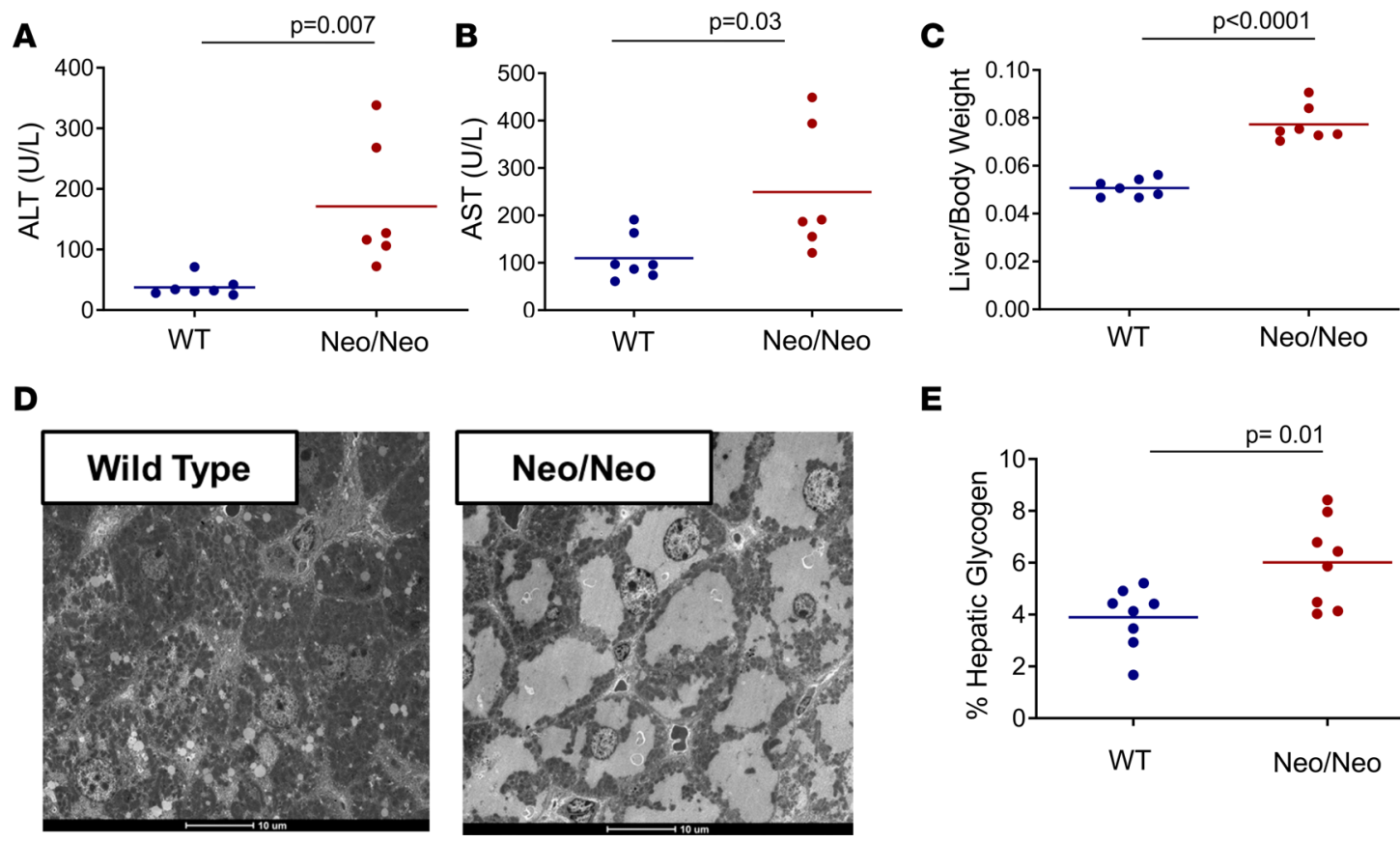

E

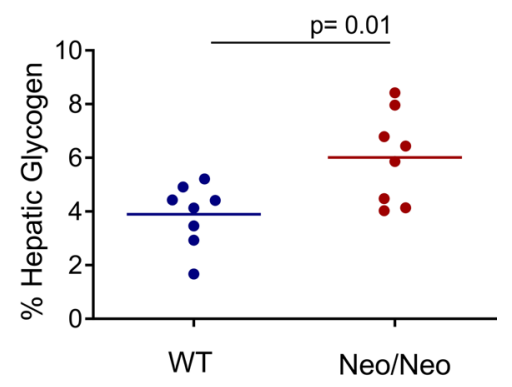

Figure 3. Hepatocellular injury and hepatic glycogen accumulation in As/ ${ }^{\text {Neo/Neo }}$ mice after 4-hour fast. (A) Elevated ALT was observed in As/Neo/Neo mice. (B) Elevated AST was observed in As/Neo/Neo mice. (C) Hepatomegaly was observed in As/Neo/Neo mice. (D) Electron microscopy demonstrates increased glycogen deposition within hepatocytes of $A s /^{\text {Neo/Neo }}$ mice, causing displacement of organelles to the cell membrane. Scale bars: $10 \mu \mathrm{m}$. (E) Quantification of hepatic glycogen in As/Neo/Neo mice versus WT mice confirms increased hepatic glycogen accumulation in As/Neo/Neo mice. For statistical comparisons, Student's independent 2-sample $t$ test was used.

were treated with helper-dependent adenovirus expressing Asl using a liver-specific promoter (liver-specific $A p o E$ genomic promoter with liver-specific enhancer) or empty virus using retro-orbital injection at approximately 2-3 weeks of age as previously described $(3,31)$. Plasma and liver samples were collected 2 weeks later after either a 3-hour fast or a 12-hour fast. Because of decreased survival of $A s^{\text {INeo/Neo mice }}$ treated with empty virus, the $A s l^{\text {Neo/Neo }}$ mice treated with helper-dependent adenovirus expressing $A s l$ were compared with historical cohorts of untreated mice.

After treatment with helper-dependent adenovirus expressing $A s l, A s l^{\text {Neo/Neo }}$ mice had significantly reduced hepatomegaly (Figure 6A) and hepatic glycogen content, whereas the enzyme activity of hepatic glycogen phosphorylase was increased (Figure 6B). There was also a trend suggesting reduced ALT (treated, mean $=53 \mathrm{U} / \mathrm{L}$ with range of $43-72 \mathrm{U} / \mathrm{L}$; untreated, mean $=171 \mathrm{U} / \mathrm{L}$ with range of 72-338 U/L, $P=0.11$ ) and AST (treated, mean $=110 \mathrm{U} / \mathrm{L}$ with range of 100-120 U/L; untreated, mean $=250 \mathrm{U} / \mathrm{L}$ with range of $121-449 \mathrm{U} / \mathrm{L}, P=0.13)$. Similarly, adeno-associated virus serotype 8 (AAV8) gene therapy has also been demonstrated to rescue the elevation in AST and ALT levels in this mouse model (27). These results suggest that rescue of the ASL deficiency in the hepatocyte rescues the chronic hepatic glycogen phenotype.

\section{Discussion}

In the largest study of individuals with UCDs to date, we demonstrate an increased burden of chronic hepatocellular injury in individuals with ASLD and ARG1D. This increased prevalence of chronic hepatocellular injury in ASLD and ARG1D is in contrast to the previously published findings describing episodic acute liver failure in OTCD that, at least in some cases, resolves with metabolic control $(32,33)$. The prevalence of chronic hepatocellular injury in individuals with ASLD of 37\% (ALT > 100 units/L) in our cohort is slightly lower than the approximately $50 \%-55 \%$ prevalence previously reported in smaller cohorts $(4,34)$. However, these cohorts were smaller in size than our cohort and used lower thresholds $(40-50 \mathrm{U} / \mathrm{L})$ to define hepatocellular injury $(4,34)$. A more recent study suggested a $90 \%$ prevalence of chronic liver disease (as assessed by AST or ALT $\geq 40 \mathrm{U} / \mathrm{L}$ over 6 months or abnormal liver ultrasound) in ASLD using a small cohort of subjects $(n=28)(20)$. Thus, we suspect 
A
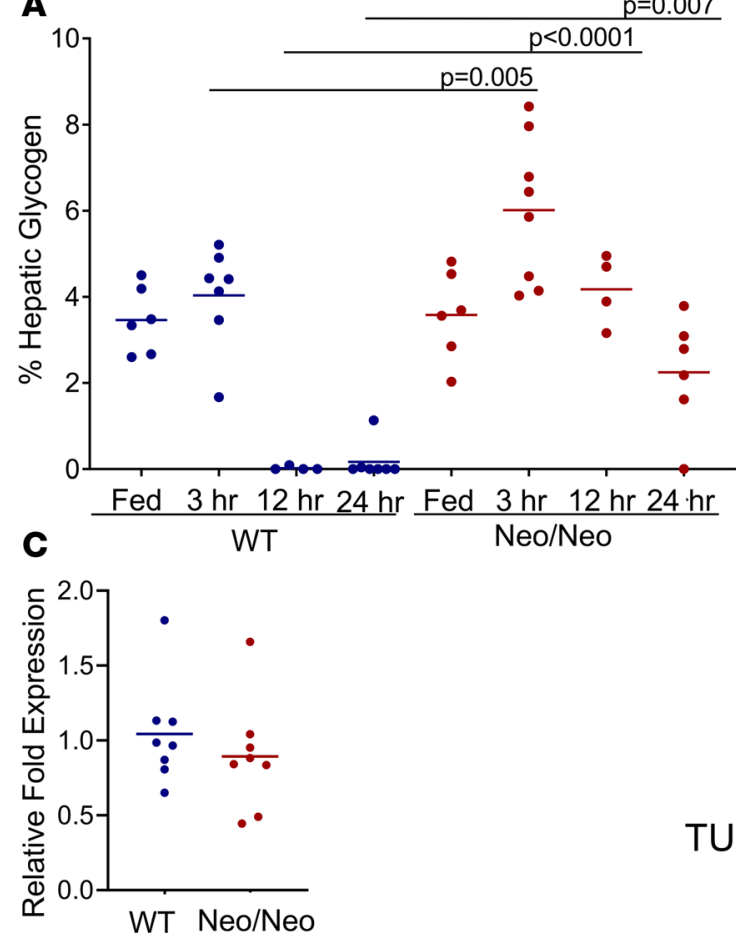

B

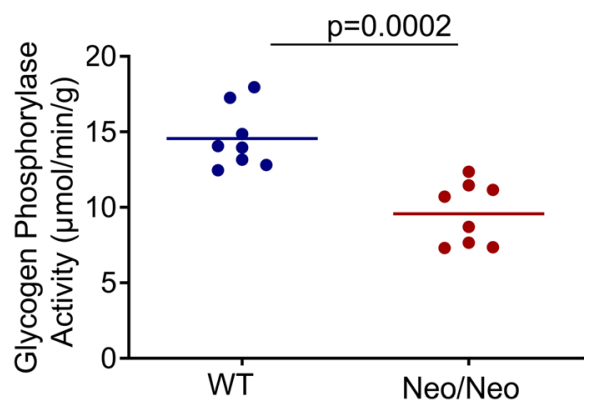

D

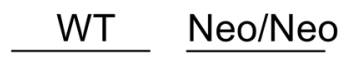

PYGL

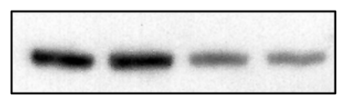

TUBULIN

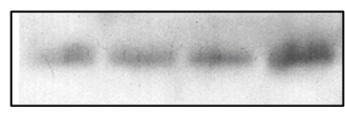

Figure 4. Impaired hepatic glycogenolysis in As/Neo/Neo mice. (A) Hepatic glycogen was quantified in WT and As/Neo/Neo mice that were not-fasted (fed) or mice that were fasted for 3, 12, or 24 hours. A 2-way ANOVA followed by Sidak's post hoc test was used to make pair-wise comparisons between WT versus As/Neo/Neo mice at each time point. (B) Hepatic glycogen phosphorylase enzyme activity is reduced in As/ ${ }^{\text {Neo/Neo }}$ mice. Student's independent 2-sample $t$ test was used. (C) Gene expression in WT and $A$ s $^{\text {Neo/Neo }}$ mice is similar. $n=8$ per genotype. (D) PYGL protein levels are reduced in As/Neo/Neo mice versus WT mice. Immunoblotting was performed with tissue from 8 WT and 8 As/Neo/Neo mice with technical replicates (representative samples shown).

that we would have detected an even higher prevalence of chronic hepatocellular injury if we used even lower thresholds for ALT and AST.

Our analysis suggests that individuals with pathogenic variants associated with severe enzyme deficiency may have increased risk for this chronic hepatocellular injury and, thus, warrant close surveillance. The chronic hepatocellular injury observed in individuals with ASLD enrolled in the Longitudinal Study of Urea Cycle Disorders and described in the literature occur in the setting of standard therapies, including low-protein diet, arginine supplementation and nitrogen-scavenging agents (4, 9, 20, 34). Similarly, standard-of-care treatment used in patients (e.g., sodium benzoate conjugation of glycine to stimulate nitrogen disposal via urinary hippurate excretion and arginine repletion therapy) did not result in normalization of ALT and/or AST levels in the mouse model of ASLD.

The long-term consequences of chronic hepatocellular injury in UCDs such as ASLD are unknown, and there are no clear management guidelines for monitoring for these complications. Moreover, hepatic biopsies are not routinely performed in individuals with UCDs; thus, hepatic histopathology is not available for most of the individuals with ASLD enrolled in our study. Therefore, chronic elevation in serum aminotransferase levels may be the only clue to underlying liver disease in many participants in our study. Hepatic glycogen accumulation and moderate to severe fibrosis and cirrhosis were noted in 3 of 4 hepatic explants available from individuals with ASLD in our local cohort. In addition, late-stage fibrosis (10, $11,15,35,36)$, cirrhosis $(12,37)$, and even hepatocellular carcinoma $(4,23)$ have been described in other individuals with ASLD, indicating that chronic hepatocellular injury may be associated with significant morbidity in at least a subset of patients. Indeed, our noninvasive biomarker study identified abnormal liver imaging or testing in a quarter of the participants with normal serum aminotransferase levels. We speculate that elevated hepatic glycogen could be contributing to the high prevalence of increased liver stiffness in the participants with ASLD, but more studies are needed to investigate this hypothesis. Alternatively, the role of hepatic steatosis (known to be a disease modifier for cirrhosis in those with obesity) in this cohort 
A

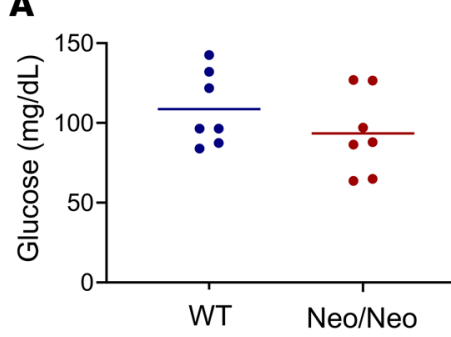

B

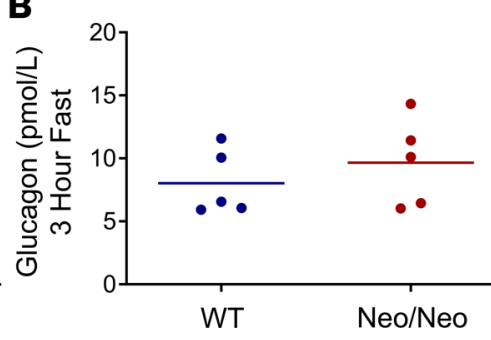

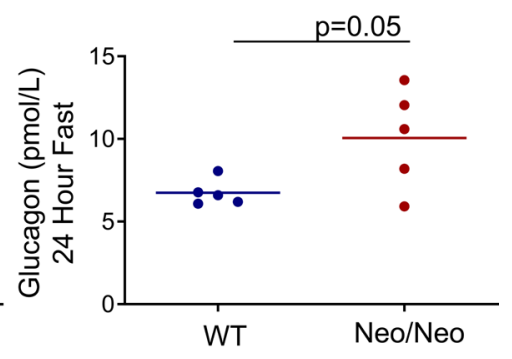

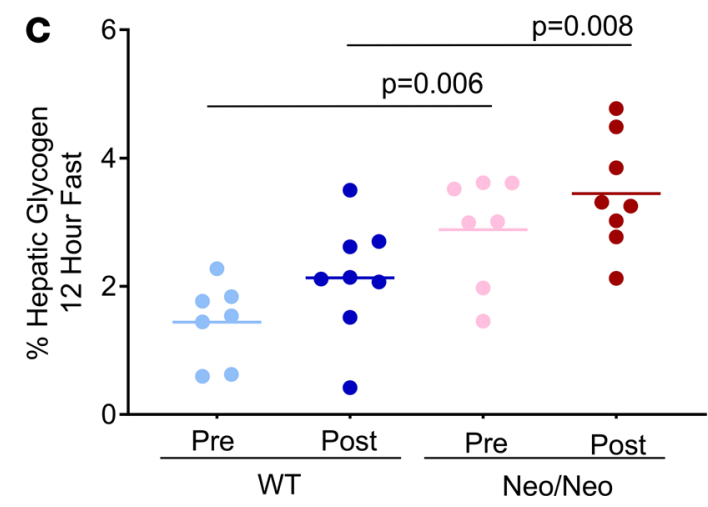

Figure 5. Plasma glucagon levels in As/Neo/Neo mice and WT mice. (A) Plasma glucose levels after a 12-hour fast (anesthetized mice used in glucose bolus study) are not significantly different between mutant and WT mice. (B) Plasma glucagon levels after a 3- and 24-hour fast are similar in As/ ${ }^{\text {Neo/Neo }}$ mice and WT mice. (C) Hepatic glycogen after a glucose bolus appears to rise similarly in As/Neo/Neo mice versus WT mice. For plasma glucagon and glucose levels,

Student's independent 2-sample $t$ test was used. For glycogen study, a 1-way ANOVA with Sidak's post hoc test was used to compare the results from the 2 genotypes before glucose bolus and in the groups after glucose bolus. Pre, after 12-hour fast but before glucose bolus; Post, 2 hours after glucose bolus.

remains unclear (38). Regardless, the results from our clinical study suggest that alternative biomarkers are needed to identify individuals with ASLD at risk for progressive liver disease, since serum levels of ALT or AST underestimate the prevalence of liver disease in this disorder.

Our results demonstrate that the $A s^{\text {Neo/Neo }}$ mouse serves as a good model for studying the hepatic complications associated with ASLD as the mice develop hepatomegaly, hepatocellular injury, and hepatic glycogen accumulation similar to humans with the disorder. Given the disease severity in the mouse model, the mice do not typically survive beyond 3-4 weeks of age (2). Although the mice do not show evidence of hepatic fibrosis at this age, it is unclear if these complications might occur at a later age if these mice had a longer lifespan. One unique feature of the hepatocellular injury in both the mouse model and in individuals with ASLD and other UCDs is hepatic glycogen accumulation. The contributions of hepatic glycogen accumulation to the chronic hepatocellular phenotype is unknown; it is also unknown whether it is a cause or consequence of the hepatocellular injury, or whether it may be independent of the hepatocellular injury. Increased hepatic glycogen is associated with hepatic complications such as hepatomegaly, fibrosis, cirrhosis, adenomas, and even hepatocellular carcinoma in hepatic glycogen storage disorders (39-43). In addition, inhibition of hepatic glycogen phosphorylase in rats causes hepatic glycogen accumulation associated with fibrosis and hepatic inflammation (44). Thus, hepatic glycogen accumulation may be contributing to the chronic hepatic complications in ASLD and possibly other UCDs. More studies are needed to investigate this hypothesis, especially since increases in hepatic glycogen are observed in several of the proximal disorders, including OTCD, which was associated with lower prevalence of chronic hepatocellular injury in the UCDC longitudinal study cohort $(15,45)$. Interestingly, this hepatic glycogen accumulation as assessed by H\&E staining in the murine model was previously rescued using an AAV8-mediated gene therapy with ubiquitous promoter (26). Similarly, we demonstrate rescue of both the hepatic glycogen accumulation and hepatic glycogen phosphorylase activity with helper-dependent adenovirus expressing $A s l$ with a hepatocyte-specific promoter and enhancer, which suggests that the phenotype may be cell autonomous.

One previous study of hepatic glycogen accumulation in UCDs suggested that the low protein diet may be contributing to this finding (45). However, our finding of altered hepatic glycogenolysis in the untreated $A s t^{\text {Neor } / \mathrm{Neo}}$ mouse fed a typical mouse chow (or murine breast milk) suggests that the hepatic glycogen accumulation may be 

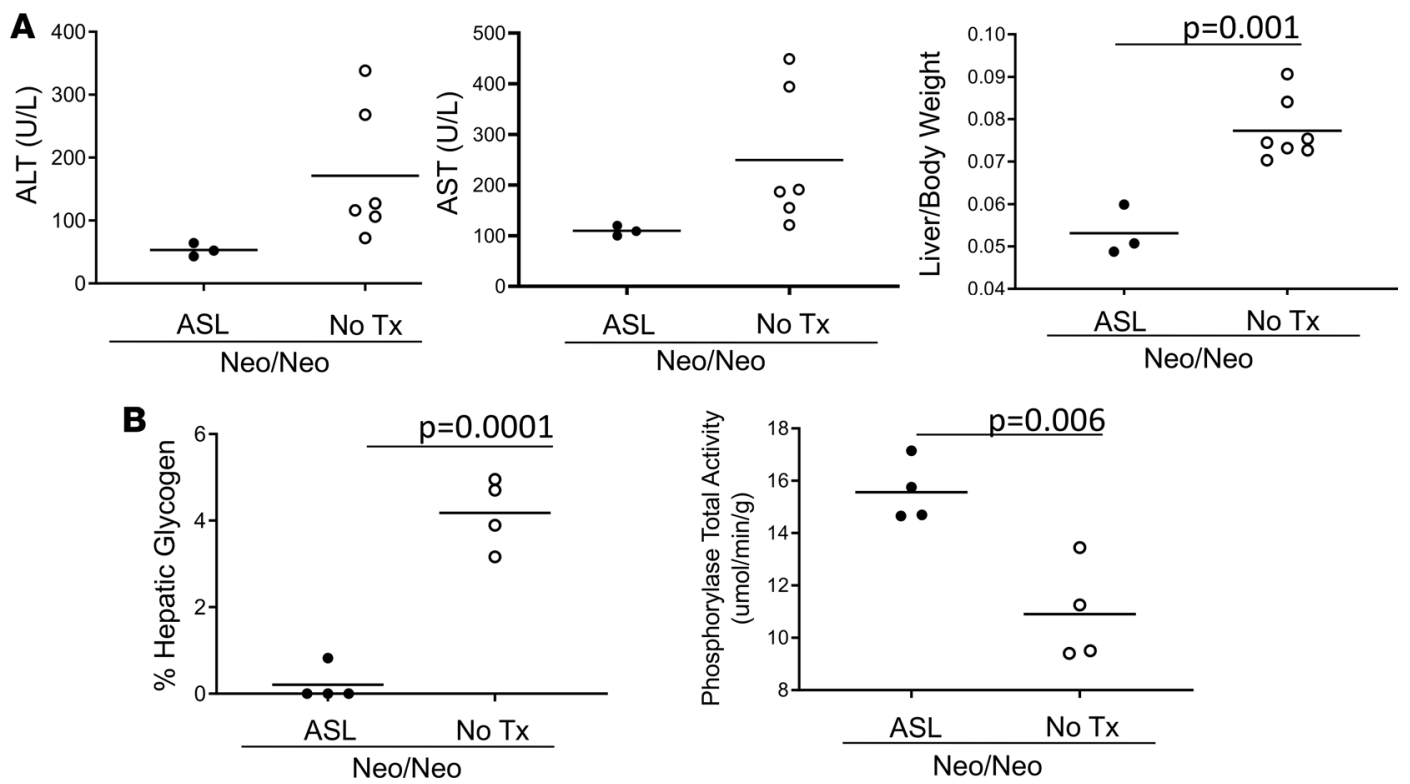

Figure 6. Helper-dependent adenovirus gene therapy (liver) corrects hepatic phenotypes in $\mathbf{A s}^{\text {Neo/Neo }}$ mice. (A) There is a trend suggesting improvement in ALT (3-hour fast, $P=0.11$ ) and AST (3-hour fast, $P=0.13$ ) after treatment with helper-dependent adenovirus expressing As/ using the ApoE promoter with liver-specific enhancer. Liver/body weight ratio was significantly improved after virus treatment. (B) Hepatic glycogen accumulation is improved and hepatic glycogen phosphorylase enzyme activity is increased after a 12-hour fast in As/ ${ }^{\mathrm{Neo} / \mathrm{Neo}}$ mice treated with virus. As/Neo/Neo mice treated with empty virus died before the end of the experiment and, thus, data from historical controls at 3-4 weeks of age are shown and used for comparison. Student's independent 2-sample $t$ test was used.

independent of the dietary and medical management of these disorders. Studies in the Asl ${ }^{\mathrm{Neo} / \mathrm{Neo}}$ mouse demonstrated a reduction in protein level and an associated reduction in activity of the hepatic glycogen phosphorylase, which catalyzes the rate-limiting step of glycogenolysis. This decrease in hepatic glycogen phosphorylase activity is specific to this enzyme, as the enzyme activities of other enzymes involved in hepatic glycogenolysis were not decreased. Interestingly, reduction in hepatic glycogen phosphorylase activity in the setting of hepatic glycogen accumulation has been observed in a liver biopsy of 1 individual with OTCD (45). The reduction of protein abundance in the absence of a comparable reduction in gene expression suggests that a posttranslation modification or other posttranscriptional regulatory mechanism results in reduced enzyme stability or increased degradation. Given the finding of NO deficiency in ASLD (10) and given that we and others have demonstrated that the hepatic glycogen phosphorylase is subject to nitrosylation (46), a posttranslational modification mediated by NO, an enticing hypothesis is that NO deficiency mediates the hepatic glycogen accumulation. However, more studies are needed to investigate this hypothesis, especially since hepatic glycogen accumulation has been observed across many UCDs, and NO deficiency appears to be a unique finding in ASLD.

Another hypothesis that has been proposed is that elevated glutamine levels stimulate insulin secretion, which contributes to hepatic glycogen accumulation (45). However, our data suggest that impaired glycogenolysis rather than increased glycogen synthesis contributes to the phenotype in our mouse model. One limitation of our study is that glycogen synthesis was assessed after a glucose bolus, and it is possible that increased glycogen synthesis occurs during the fasting period, which could result in increased glycogen cycling. Alternatively, perhaps, generalized urea cycle dysfunction through a yet-unidentified mechanism contributes to the altered glycogen metabolism in UCDs. Lastly, an impairment of glycophagy might also explain the phenotype. Further studies are needed to investigate these hypotheses.

Interestingly, the prevalence of chronic hepatocellular injury in ARG1D was nearly as high as in ASLD, although the sample size for ARG1D was considerably smaller than ASLD. Interestingly, individuals with ARG1D also had a high prevalence of elevations in INR. Thus, further studies in larger cohorts of individuals with ARG1D are needed to confirm these findings.

Given the evidence for liver disease in ASLD (and possibly other UCDs), providers should issue anticipatory guidance regarding liver health to individuals with this disorder (47), namely avoidance of alcohol, promotion of a healthy body weight, and avoidance of hepatotoxic medications. Moreover, we suggest providers ensure adequate vaccination and immunity against hepatotropic viruses, such as hepatitis $A$ and $B$, in patients with ASLD. 
Lastly, surveillance for portal hypertension (e.g., splenomegaly, declining platelet counts) should be considered and surveillance for hepatocellular carcinoma offered in individuals with advanced fibrosis or cirrhosis.

Our studies have several potential limitations. First, our analysis of the longitudinal study data only included data from study visits, and not from hospitalizations; thus, an accurate prevalence of acute liver failure in each disorder could not be determined. Moreover, we used ALT and AST as surrogate markers of chronic liver disease. Because creatine kinase (CK) levels are not routinely collected during the longitudinal study visits, we are unable to distinguish AST and ALT elevations that arise from muscle disease versus liver disease. Moreover, the follow-up time for each participant varied. In addition, comparisons of the histopathology were limited by the overrepresentation of infant males with OTCD. For the clinical study, SWE measures liver stiffness, which we presume reflects fibrosis but could represent vascular congestion or other etiologies; it cannot be confirmed to represent scarring in many of the subjects. Moreover, we use manufacturer values for defining increased liver stiffness, which were validated in adult cohorts even though our ASLD study cohort consists of participants of varied ages. Furthermore, hepatic steatosis causes the liver to be soft, so elevated liver stiffness in patients with steatosis may represent true fibrotic pathology. Lastly, for our murine studies, our mouse model only survives until 3-4 weeks of age, and this short lifespan limits our ability to study long-term liver disease in this model.

Overall, we demonstrate that chronic hepatocellular injury is a complication in individuals with UCDs, especially ASLD. However, measurement of serum hepatic aminotransferases likely underestimates the true prevalence of chronic liver disease and hepatic glycogen accumulation in individuals with this disorder. Thus, our study reiterates the need for exploring potentially new biomarkers for liver disease in ASLD and other UCDs. Clinical approaches used in the management of chronic liver disease should be considered for individuals with ASLD. The liver disease in ASLD in both humans and mice is associated with excessive glycogen accumulation, and investigations of the mouse model indicate an impairment in hepatic glycogen metabolism. Our results demonstrate a link between urea cycle dysfunction and impaired hepatic glucose metabolism and identify the ASLD mouse as an important model for further studies of liver disease in the setting of urea cycle dysfunction.

\section{Methods}

\section{Human studies}

Longitudinal study. Data from participants enrolled in the Longitudinal Study of Urea Cycle Disorders (ClinicalTrials.gov NCT00237315), a natural history study conducted by the NIH RDCRN UCDC, was obtained (28). Data from baseline visits and the semiannual or annual longitudinal study visits collected between March 1, 2006, and July 31, 2014, were used for this analysis. At the time of data capture, individuals with UCDs were enrolled at 14 sites in USA, Canada, and Europe. Data were collected at each center in a standard format, as outlined by the manual of operations using electronic case report forms and were managed by the Data Management and Coordinating Center of the RDCRN. Typically, at the time of these longitudinal study visits, participants are at their baseline state of health. For participants who received liver transplantation, only data before liver transplantation were used for this analysis.

For this analysis, serum ALT, serum AST, and INR from baseline and longitudinal study visits were used. For INR, any entry with a value entered of "0" was removed from the analysis, as this was assumed to be a data entry error. A general linear mixed model was used to compare ALT, AST, and INR between disorders. ALT, AST, and INR were transformed using a natural logarithm for analysis. The regression model included fixed effects for age at enrollment, UCD subtype, time (continuous), and a disorder-time interaction term. The baseline or enrollment visit was assumed to be time 0 for each participant. The model also included a random intercept and slope to model repeated measures within an individual over time. Statistical significance for model coefficients was assessed at the 0.05 level. Pairwise differences between disorders were assessed at the adjusted 0.05 level using Tukey's method. Serum ALT and AST for individuals with ASLD were analyzed using linear regression to assess for an association between log-transformed ALT and AST with covariates at baseline. The following covariates were analyzed: baseline age; sex; history of hyperammonemia (yes or no); baseline levels of serum arginine, citrulline, and glutamine; use of sodium benzoate (yes or no); use of sodium or glycerol phenylbutyrate (yes or no); use of sodium benzoate, sodium phenylbutyrate, or glycerol phenylbutyrate (yes or no); and use of high dose arginine as defined by a dose $\geq 5 \mathrm{~g} / \mathrm{m}^{2} /$ day if weight is $>20 \mathrm{~kg}$ or a dose of $250 \mathrm{mg} / \mathrm{kg} /$ day if weight is under $20 \mathrm{~kg}(48)$. 
Clinical histopathology study. A retrospective chart review was performed to identify all individuals with UCDs who had liver transplantation at Texas Children's Hospital from January 1, 1995, to July 1, 2016. All explant slides were reviewed for hepatic glycogen deposition, for fibrosis or cirrhosis, and for other histopathologic abnormalities using standard clinical staining procedures. All samples were scored for degree of fibrosis using the scoring system previously published by Kleiner et al. (30).

Clinical biomarker study. The study was performed at Texas Children's Hospital as part of a larger study evaluating biomarkers in participants with various UCDs. Only data from individuals with ASLD are presented here. The inclusion criteria for enrollment were age greater than or equal to 5 years and age less than or equal to 60 years, weight greater than or equal to $11 \mathrm{~kg}$, and a diagnosis of ASLD based on biochemical, molecular, or enzymatic testing. Individuals were excluded if they had a history of hyperammonemia (blood ammonia greater than 100 micromoles/L) documented in the medical record or reported by the participant in the 30 days preceding enrollment visit, history of liver transplantation, current pregnancy, or confirmed diagnosis of chronic viral hepatitis, autoimmune liver disease, or alcohol-related liver disease.

Blood was collected after a 4-hour fast for routine hepatic labs and biomarker studies. Serum was submitted to Quest Diagnostics for FibroTest assessment. FibroTest scores are calculated using the patient age, sex, $\alpha 2$-macroglobulin, haptoglobin, apolipoprotein A1, and $\gamma$-glutamyl transpeptidase and have been demonstrated to correlate with degree of hepatic fibrosis on liver biopsy in various populations (49). SWE was also performed after a 4-hour fast within 2 weeks of the blood sample collection. The abdominal ultrasound with SWE were performed on a GE Logiq E9 ultrasound equipment by a trained technologist. Each ultrasound exam consisted of standard grayscale examination (with measurement) of the liver, pancreas, spleen, and all 4 quadrants and a standard Doppler interrogation of the relevant hepatic and splenic vasculature. In addition, SWE measurements of the hepatic segments 5 and 8 (5-6 measurements per segment) were acquired using a right intercostal approach. Measurements were obtained using a curvilinear transducer per the standard department protocol. For the purposes of this study, the standard manufacturer's cutoffs for fibrosis were used (50) as follows: F > 0,1.35 m/s; F > 1, $1.66 \mathrm{~m} / \mathrm{s} ; \mathrm{F}>2,1.77 \mathrm{~m} / \mathrm{s} ; \mathrm{F}>3,1.99 \mathrm{~m} / \mathrm{s}$; and F $>4,2.2 \mathrm{~m} / \mathrm{s}$. Of note, these cutoffs were determined using measurements from adults with hepatitis $\mathrm{C}(50)$.

\section{Murine studies}

Mouse colony management. The generation of the $A s l^{\text {Neo/Neo }}$ mice used in these studies was previously described, and the colony was maintained by intercrossing heterozygous animals (2). The mouse colony was housed in the Baylor College of Medicine Transgenic Mouse Facility, and male and female mice were used for all studies. The mice were maintained in microisolator cages within ventilated racks in rooms with a 12-hour/12-hour, light/dark cycle. Mice were fed Lab Diet 5V5R or Envigo Teklad 2020 chows, which have comparable nutritional content and provided autoclaved water. For fasting studies, food was removed and mice were placed in clean cages with drinking water starting at $7 \mathrm{am}$ for the time course indicated for individual studies (unless otherwise specified). For double-therapy treatment studies, we treated mice with sodium benzoate $(250 \mathrm{mg} / \mathrm{kg}$ per day, Spectrum Chemical) and with L-arginine free base (100 mg/kg per day, Thermo Fisher Scientific) mixed in the drinking water as previously described (2). The treated drinking water was provided to the mother's cage before delivery and throughout development until the time of study. Doses are estimates based on average water consumption.

Blood chemistry and tissue collection. For blood collection, mice were anesthetized with isoflurane, and retro-orbital collections were performed. Plasma was collected and stored at $-80^{\circ} \mathrm{C}$ until analysis. All liver samples were collected after euthanasia and snap-frozen in liquid nitrogen for glycogen analysis. Blood chemistry including AST, ALT, and direct bilirubin were performed using standard procedures by the Center for Comparative Medicine Comparative Pathology Laboratory at Baylor College of Medicine. Histology and electron microscopy were performed on sections of liver collected by the Texas Medical Center Digestive Diseases Center Cellular and Molecular Morphology Core. Glucagon was measured using ELISA (Mercodia). Plasma glucose was assessed using the liquid glucose oxidase reagent set (Pointe Scientific).

Glycogen enzyme activity and glycogen content studies. Phosphorylase (1,4- $\alpha$-D-glucan; phosphate $\alpha$-D-glucosyltransferase; EC 2.4.I.I) activity was analyzed in snap-frozen liver tissue using a standard spectrophotometric methods (51-53). The enzyme activity was assayed indirectly by measuring the amount of free phosphate released using inorganic phosphorous reagent (ClearChem Diagnostics), and the activity was expressed as $\mu \mathrm{mol} / \mathrm{min} / \mathrm{g}$. Activity of the phosphorylase kinase and debranching enzyme was measured using published protocols (53, 54). Branching enzyme activity was measured in frozen liver tissue using standard spectrophotometric methods. 
The residual enzyme activity was assayed indirectly by measuring the amount of phosphate released using Roche phosphate reagent, and the activity was expressed as $\mu \mathrm{mol} / \mathrm{min} / \mathrm{g}$ liver tissue $(55,56)$. Glycogen content was measured indirectly by the release of free glucose from glucose reagent (Thermo Fisher Scientific) using a standard protocol. Enzyme and glycogen content studies were performed blinded to genotype.

Protein and RNA expression studies. RNA was generated from liver tissue using the Qiagen All Prep DNA/RNA/Protein kit (catalog number 80004). cDNA was generated by reverse transcription using the Superscript III First Strand kit from Thermo Fisher Scientific (catalog 18080-051). Quantitative PCR was performed to assess mouse liver glycogen phosphorylase ( $P y g l$ ) gene expression using primers $5^{\prime}$-TTCCAGCCACAGACCTATCGGAGCAG-3' (forward) and 5'-CGTAATATTCTTTGGCCTCATACCCCTT-3' (reverse) and an annealing temperature of $60^{\circ} \mathrm{C}$. Gene expression of $B 2 m$ was used for comparison (e.g, housekeeping gene) with the following primers used: 5'-GGTCTTTCTGGTGCTTGTC-3' (forward) and 5'-CGTATGTATCAGTCTCAGT-3' (reverse) with annealing temperature of $65^{\circ} \mathrm{C}$.

Western blot was performed using standard techniques to assess protein levels. Briefly, lysates were generated from approximately $100 \mathrm{mg}$ of tissue in $500 \mu \mathrm{L}$ of Pierce RIPA buffer (Thermo Fisher Scientific, catalog 89900) using a handheld homogenizer (or TissueLyser II, Qiagen). Protein concentration was measured using the microBCA assay kit from Thermo Fisher Scientific (catalog 23235). The blot was run by loading $30 \mu \mathrm{g}$ of each sample on a 10\% gel from Bio-Rad (catalog 4561034) followed by transfer of proteins to a PVDF membrane. Polycolonal antibody to glycogen phosphorylase (PYGL), liver isoform (dilution 1:1000) from ProteinTech (catalog 15851-1-AP) was used as the primary antibody, and HRP conjugated anti-rabbit antibody from GE Healthcare (catalog NA934V) was used as the secondary antibody. Protein detection was performed with Luminata Crescendo Western HRP substrate from MilliporeSigma (catalog WBLUR0100). Monoclonal anti- $\alpha$-tubulin (primary antibody, MilliporeSigma, T5168; secondary antibody, Bio-Rad, anti-mouse, catalog 1721011) was used to control for protein loading. Immunoblotting for the hepatic glycogen phosphorylase (untreated mice) was performed on a total of $8 \mathrm{WT}$ and $8 \mathrm{As} l^{\text {Neo/Neo }}$ mice with technical replicates, and representative samples are shown in Figure 4D.

Helper-dependent adenovirus studies. For liver-specific gene therapy rescue studies, the helper-dependent adenoviral vector (HDAd-gE-mAsl) containing $m A s l$ expressed under the control of the liver-specific ApoE genomic promoter with liver-specific enhancer has been previously described $(3,31)$. The same helper-dependent adenoviral vector backbone containing the phosphorylase gene (HDAd-gE-mPygl) was used for liver-specific overexpression of the glycogen phosphorylase enzyme. Empty virus was used as control. Helper-dependent adenovirus was delivered $\left(5 \times 10^{12} \mathrm{vp}\right.$ per $\left.\mathrm{kg}\right)$ with a single retro-orbital injection in mice that were anesthetized with isoflurane. For helper-dependent adenovirus studies, the $A s^{l^{N e o} / N e o}$ mice remained with the dam until the study was completed because these mice typically do not survive weaning (2). Moreover, the mutant mice appear to consume breast milk after 3 weeks of age; milk has been identified in the stomachs between 3-5 weeks of age. For fasting studies, the mice were separated from the dam.

Hepatic glycogen synthesis study. Mice were fasted 12 hours starting the evening before the procedure. After the 12-hour fast, 1 cohort of mice was anesthetized with isoflurane, and blood was collected (retro-orbital) for plasma glucose measurements. Then, liver tissue was harvested for hepatic glycogen quantification. In a second cohort of mice, the mice were given a $20 \%$ glucose solution via an i.p. injection (dose of 2 grams of glucose per kilogram of body weight), and fasting was continued for 2 additional hours. Two hours after glucose bolus was given, the mice were anesthetized with isoflurane for blood collection. Then, the mice were euthanized for collection of liver tissue. Liver tissue was snap-frozen in liquid nitrogen and stored at $-80^{\circ} \mathrm{C}$ until hepatic glycogen quantification was performed. To determine the quantity of liver glycogen in liver samples for this hepatic glycogen synthesis study only, the liver samples were homogenized in double deionized water. One aliquot of each sample was mixed with $6 \mathrm{~N} \mathrm{HCl}(2: 1$ ratio) in order to hydrolyze the glycogen and was boiled for 2 hours; then, an equivalent volume of $6 \mathrm{~N} \mathrm{NaOH}$ was added. Glucose in the sample was then measured using the Cayman glucose kit (Cayman Chemical) as per manufacturer's instructions in order to determine the total glucose (glycogen + free glucose) in the liver homogenate. Similarly, to determine the free glucose in the sample, a second aliquot of each sample was mixed with $6 \mathrm{~N} \mathrm{NaOH}(2: 1$ ratio) and was boiled for 2 hours; then, an equivalent volume of $6 \mathrm{~N} \mathrm{HCl}$ was added. Glucose measurements were made using Cayman glucose kit (Cayman Chemical) to determine the free glucose in the liver homogenate. To calculate the quantity of glycogen in the sample, the free glucose was subtracted from the total glucose.

Statistics. Statistical analysis methods for the longitudinal data was performed using Stata v 12.1 (Stata Corp.) and are described above. For all other statistical analyses, GraphPad Prism v.8.1.1 was used. For the 
animal studies, Student's independent 2-tailed, 2-sample $t$ test was used to compare 2 groups. For comparing multiple groups, either a 1- or 2-way ANOVA was used followed by Sidak's post hoc test for comparing selected groups. For correlation studies, a Pearson correlation coefficient was used. $P<0.05$ was used for all studies.

Study approval. The study protocol for the Longitudinal Study of Urea Cycle Disorders was approved by the NICHD, and the study is being monitored by a data safety monitoring board of the NICHD. Between 2007 and 2015, the study procedures were reviewed and approved by the IRBs of all participating clinical sites. From 2015, the study was conducted under a protocol that was approved by the IRB of the Children's National Medical Center (Washington, DC, USA) which now serves as the central IRB of record for the UCDC. Written informed consent was obtained from all participants or their legal guardians.

The elastography and serum biomarker study was conducted under a protocol that was approved by the IRB of the Children's National Medical Center. Written informed consent was obtained for all participants or their legal guardians. The histopathology review study was approved as a retrospective chart review by the Baylor College of Medicine IRB. The Baylor College of Medicine IACUC approved all mouse studies.

\section{Author contributions}

LCB designed the human studies and mouse experiments, acquired data from the mouse model, and analyzed and interpreted data from the human and mouse studies. LCB supervised the data collection and interpretation of all human studies. LCB is listed as the first co-first author because she participated in the mouse studies, led the human studies, and led the preparation of the final draft of the manuscript, with assistance from SM. SM participated in the design of the mouse studies, conducted mouse experiments, analyzed and interpreted data from the mouse studies, and assisted with the preparation of the first draft of the manuscript. XL and BMS conducted experiments with the mouse model and managed the mouse colony. SA coordinated the elastography and serum biomarker study. MM and JM conducted experiments and participated in data analysis and interpretation in the mouse model. MMJ, RC, and TB conducted experiments with the mouse model. ZJ assisted with the western blot studies. JD and DB conducted the murine hepatic glycogen quantification and enzyme analysis and assisted in data interpretation. DG and CGM performed the statistical analysis and interpretation of the human longitudinal study data. MF and DS performed and interpreted the histopathology of the mouse and human liver samples. Members of the UCDC acquired longitudinal study data. SN assisted in the statistical analysis and interpretation of the longitudinal study data and assisted with design and recruitment for the elastography and serum biomarker study. PM designed, performed, and interpreted the ultrasounds with shear-wave elastography. BLS and DHL assisted with design and interpretation of the ultrasound with shear-wave elastography and biomarker study. BL supervised the data collection and interpretation of all experiments using the mouse model. Members of the UCDC designed and collected data for the Longitudinal Study of Urea Cycle Disorders. All authors participated in the editing of the manuscript and approved final version of the manuscript.

\section{Acknowledgments}

We thank the participants and families for their participation. This work was supported by the Genzyme/ ACMG Foundation for Genetic and Genomic Medicine Medical Genetics Training Award in Clinical Biochemical Genetics (LCB), the National Urea Cycle Disorders Foundation Fellowship (LCB), a fellowship from the UCDC (U54HD061221, LCB) K08DK106453 (LCB), and the US Public Health Service grant P30DK56338, which funds the Texas Medical Center Digestive Diseases Center. LCB is also the recipient of a Burroughs Wellcome Fund Career Award for Medical Scientists. This work was also supported by the R01DK102641 (BL) and the Baylor College of Medicine Intellectual Intellectual and Developmental Disabilities Research Center (HD024064) from the Eunice Kennedy Shriver National Institute of Child Health and Human Development (NICHD). SM was supported by Howard Hughes Med into Grad Scholarship awarded through the Translational Biology and Molecular Medicine Graduate Program at Baylor College of Medicine and a predoctoral fellowship from the Cancer Prevention and Research Institute of Texas. BMS was supported by NIH T32DK007664-28 and the US Public Health Service grant P30DK56338, which funds the Texas Medical Center Digestive Diseases Center.

The UCDC (U54HD061221) is a part of the NIH RDCRN, supported through collaboration between the Office of Rare Diseases Research (ORDR), the National Center for Advancing Translational Science (NCATS), the Eunice Kennedy Shriver NICHD, and the National Institute of Diabetes and Digestive 
and Kidney Diseases (NIDDK). The UCDC is also supported by the O'Malley Foundation, Rotenberg Family Fund, Dietmar-Hopp Foundation, Kettering Fund, and National Urea Cycle Disorders Foundation. DG was supported by Clinical Translational Core of the Baylor College of Medicine IDDRC (1 U54 HD083092) from the Eunice Kennedy Shriver NICHD. Please see Supplemental Acknowledgments for consortium details. We would also like to thank Denise Peterson for her assistance with glycogenosis enzyme analysis of murine liver tissue.

Address correspondence to: Lindsay C. Burrage, Department of Molecular and Human Genetics, Baylor College of Medicine, One Baylor Plaza, Room R807, Houston, Texas 77030, USA. Phone: 832.822.4280; Email: burrage@bcm.edu. Or to: Brendan Lee, Department of Molecular and Human Genetics, Baylor College of Medicine, One Baylor Plaza, Room R814, Houston, Texas 77030, USA. Phone: 713.798.8853; Email: blee@bcm.edu.

1. Shih VE, Littlefield JW, Moser HW. Argininosuccinase deficiency in fibroblasts cultured from patients with argininosuccinic aciduria. Biochem Genet. 1969;3(1):81-83.

2. Erez A, et al. Requirement of argininosuccinate lyase for systemic nitric oxide production. Nat Med. 2011;17(12):1619-1626.

3. Nagamani SC, et al. Nitric-oxide supplementation for treatment of long-term complications in argininosuccinic aciduria. Am J Hum Genet. 2012;90(5):836-846.

4. Baruteau J, et al. Expanding the phenotype in argininosuccinic aciduria: need for new therapies. J Inherit Metab Dis. 2017;40(3):357-368.

5. Brunetti-Pierri N, Erez A, Shchelochkov O, Craigen W, Lee B. Systemic hypertension in two patients with ASL deficiency: a result of nitric oxide deficiency? Mol Genet Metab. 2009;98(1-2):195-197.

6. Fakler CR, Kaftan HA, Nelin LD. Two cases suggesting a role for the L-arginine nitric oxide pathway in neonatal blood pressure regulation. Acta Paediatr. 1995;84(4):460-462.

7. Kho J, et al. Argininosuccinate Lyase Deficiency Causes an Endothelial-Dependent Form of Hypertension. Am J Hum Genet. 2018;103(2):276-287.

8. Brambilla A, et al. Resting energy expenditure in argininosuccinic aciduria and in other urea cycle disorders. J Inherit Metab Dis. 2019;42(6):1105-1117.

9. Tuchman M, et al. Cross-sectional multicenter study of patients with urea cycle disorders in the United States. Mol Genet Metab. 2008;94(4):397-402.

10. Mori T, et al. Progressive liver fibrosis in late-onset argininosuccinate lyase deficiency. Pediatr Dev Pathol. 2002;5(6):597-601.

11. Zimmermann A, Bachmann C, Baumgartner R. Severe liver fibrosis in argininosuccinic aciduria. Arch Pathol Lab Med. 1986;110(2):136-140.

12. Marble M, et al. Living related liver transplant in a patient with argininosuccinic aciduria and cirrhosis: metabolic follow-up. J Pediatr Gastroenterol Nutr. 2008;46(4):453-456.

13. Parsons HG, Scott RB, Pinto A, Carter RJ, Snyder FF. Argininosuccinic aciduria: long-term treatment with arginine. $J$ Inherit Metab Dis. 1987;10(2).

14. Martín-Hernández E, et al. Urea cycle disorders in Spain: an observational, cross-sectional and multicentric study of 104 cases. Orphanet J Rare Dis. 2014;9:187.

15. Yaplito-Lee J, Chow CW, Boneh A. Histopathological findings in livers of patients with urea cycle disorders. Mol Genet Metab. 2013;108(3):161-165.

16. Trevisson E, et al. Argininosuccinate lyase deficiency: mutational spectrum in Italian patients and identification of a novel ASL pseudogene. Hum Mutat. 2007;28(7):694-702.

17. Al-Sayed M, et al. Identification of a common novel mutation in Saudi patients with argininosuccinic aciduria. J Inherit Metab Dis. 2005;28(6):877-883.

18. Kölker S, et al. The phenotypic spectrum of organic acidurias and urea cycle disorders. Part 2: the evolving clinical phenotype. $J$ Inherit Metab Dis. 2015;38(6):1059-1074.

19. Bigot A, Tchan MC, Thoreau B, Blasco H, Maillot F. Liver involvement in urea cycle disorders: a review of the literature. J Inherit Metab Dis. 2017;40(6):757-769.

20. Ranucci G, et al. Chronic liver involvement in urea cycle disorders. J Inherit Metab Dis. 2019;42(6):1118-1127.

21. Travers H, Reed JS, Kennedy JA. Ultrastructural study of the liver in argininosuccinase deficiency. Pediatr Pathol. 1986;5(3-4):307-318.

22. Badizadegan K, Perez-Atayde AR. Focal glycogenosis of the liver in disorders of ureagenesis: its occurrence and diagnostic significance. Hepatology. 1997;26(2):365-373.

23. Wilson JM, Shchelochkov OA, Gallagher RC, Batshaw ML. Hepatocellular carcinoma in a research subject with ornithine transcarbamylase deficiency. Mol Genet Metab. 2012;105(2):263-265.

24. Koo M, Lipshutz GS, Cederbaum SD, Lassman C. Biopsy-proven Hepatocellular Carcinoma in a 53-year-old Woman With Arginase Deficiency. Pediatr Dev Pathol. 2017;20(6):517-521.

25. Tsang JP, et al. Arginase deficiency with new phenotype and a novel mutation: contemporary summary. Pediatr Neurol. 2012;47(4):263-269.

26. Baruteau J, et al. Argininosuccinic aciduria fosters neuronal nitrosative stress reversed by Asl gene transfer. Nat Commun. 2018;9(1):3505.

27. Ashley SN, Nordin JML, Buza EL, Greig JA, Wilson JM. Adeno-associated viral gene therapy corrects a mouse model of argininosuccinic aciduria. Mol Genet Metab. 2018;125(3):241-250. 
28. Batshaw ML, Tuchman M, Summar M, Seminara J, Members of the Urea Cycle Disorders Consortium. A longitudinal study of urea cycle disorders. Mol Genet Metab. 2014;113(1-2):127-130.

29. Balmer C, et al. Mutations and polymorphisms in the human argininosuccinate lyase (ASL) gene. Hum Mutat. 2014;35(1):27-35.

30. Kleiner DE, et al. Design and validation of a histological scoring system for nonalcoholic fatty liver disease. Hepatology. 2005;41(6):1313-1321.

31. Kim IH, Józkowicz A, Piedra PA, Oka K, Chan L. Lifetime correction of genetic deficiency in mice with a single injection of helper-dependent adenoviral vector. Proc Natl Acad Sci USA. 2001;98(23):13282-13287.

32. Gallagher RC, Lam C, Wong D, Cederbaum S, Sokol RJ. Significant hepatic involvement in patients with ornithine transcarbamylase deficiency. J Pediatr. 2014;164(4):720-725.e6.

33. Laemmle A, et al. Frequency and Pathophysiology of Acute Liver Failure in Ornithine Transcarbamylase Deficiency (OTCD). PLoS ONE. 2016;11(4):e0153358.

34. AlTassan R, Bubshait D, Imtiaz F, Rahbeeni Z. A retrospective biochemical, molecular, and neurocognitive review of Saudi patients with argininosuccinic aciduria. Eur J Med Genet. 2018;61(6):307-311.

35. Yankol Y, Mecit N, Kanmaz T, Acarli K, Kalayoglu M. Argininosuccinic Aciduria-A Rare Indication for Liver Transplant: Report of Two Cases. Exp Clin Transplant. 2017;15(5):581-584.

36. Newnham T, et al. Liver transplantation for argininosuccinic aciduria: clinical, biochemical, and metabolic outcome. Liver Transpl. 2008;14(1):41-45.

37. Özçay F, Barış Z, Moray G, Haberal N, Torgay A, Haberal M. Report of 3 Patients With Urea Cycle Defects Treated With Related Living-Donor Liver Transplant. Exp Clin Transplant. 2015;13 Suppl 3:126-130.

38. Kabbany MN, et al. Prevalence of Nonalcoholic Steatohepatitis-Associated Cirrhosis in the United States: An Analysis of National Health and Nutrition Examination Survey Data. Am J Gastroenterol. 2017;112(4):581-587.

39. Göğüş S, et al. Histologic features of the liver in type Ia glycogen storage disease: comparative study between different age groups and consecutive biopsies. Pediatr Dev Pathol. 2002;5(3):299-304.

40. Kishnani PS, et al. Diagnosis and management of glycogen storage disease type I: a practice guideline of the American College of Medical Genetics and Genomics. Genet Med. 2014;16(11):e1

41. Roscher A, et al. The natural history of glycogen storage disease types VI and IX: Long-term outcome from the largest metabolic center in Canada. Mol Genet Metab. 2014;113(3):171-176.

42. Tsilianidis LA, et al. Aggressive therapy improves cirrhosis in glycogen storage disease type IX. Mol Genet Metab. 2013;109(2):179-182.

43. Halaby CA, et al. Liver fibrosis during clinical ascertainment of glycogen storage disease type III: a need for improved and systematic monitoring. Genet Med. 2019;21(12):2686-2694.

44. Floettmann E, et al. Prolonged inhibition of glycogen phosphorylase in livers of Zucker Diabetic Fatty rats models human glycogen storage diseases. Toxicol Pathol. 2010;38(3):393-401.

45. Miles L, Heubi JE, Bove KE. Hepatocyte glycogen accumulation in patients undergoing dietary management of urea cycle defects mimics storage disease. J Pediatr Gastroenterol Nutr. 2005;40(4):471-476.

46. Lee TY, et al. dbSNO: a database of cysteine S-nitrosylation. Bioinformatics. 2012;28(17):2293-2295.

47. Baruteau J, et al. Argininosuccinic aciduria: Recent pathophysiological insights and therapeutic prospects. J Inherit Metab Dis. 2019;42(6):1147-1161.

48. Nagamani SC, et al. A randomized controlled trial to evaluate the effects of high-dose versus low-dose of arginine therapy on hepatic function tests in argininosuccinic aciduria. Mol Genet Metab. 2012;107(3):315-321.

49. Myers RP, Ratziu V, Imbert-Bismut F, Charlotte F, Poynard T, MULTIVIRC Group. Groupe d'Etude Multidisciplinaire sur les Pathologies Liées au Virus C. Biochemical markers of liver fibrosis: a comparison with historical features in patients with chronic hepatitis C. Am J Gastroenterol. 2002;97(9):2419-2425.

50. Barr RG. Elastography Assessment of Liver Fibrosis: Society of Radiologists in Ultrasound Consensus Conference Statement. Radiology. 2015;276(3):845-861.

51. Lederer B, Van Hoof F, Van den Berghe G, Hers H. Glycogen phosphorylase and its converter enzymes in haemolysates of normal human subjects and of patients with type VI glycogen-storage disease. A study of phosphorylase kinase deficiency. Biochem J. 1975;147(1):23-35.

52. Lederer B, Stalmans W. Human liver glycogen phosphorylase. Kinetic properties and assay in biopsy specimens. Biochem J. 1976;159(3):689-695.

53. Brown BI, Brown DH. Definitive assays for glycogen debranching enzyme in human fibroblasts. In: Schotland DL, ed. Disorders of the motor unit. New York, New York, USA: J. Wiley; 1982:667-673.

54. Tuchman M, Brown BI, Burke BA, Ulstrom RA. Clinical and laboratory observations in a child with hepatic phosphorylase kinase deficiency. Metab Clin Exp. 1986;35(7):627-633.

55. Brown DH, Brown BI. Studies of the residual glycogen branching enzyme activity present in human skin fibroblasts from patients with type IV glycogen storage disease. Biochem Biophys Res Commun. 1983;111(2):636-643.

56. L'herminé-Coulomb A, et al. Fetal type IV glycogen storage disease: clinical, enzymatic, and genetic data of a pure muscular form with variable and early antenatal manifestations in the same family. Am J Med Genet A. 2005;139A(2):118-122. 